\title{
Azilsartan causing metabolic encephalopathy
}

\author{
Anujith G. Sekhar ${ }^{1}$, Ajit Singh ${ }^{2}$, Sheetal Chauhan ${ }^{3}$, Kanav Khera $^{1 *}$, Tom Devasia ${ }^{2}$ \\ ${ }^{1}$ Department of Pharmacy Practice, Manipal College of Pharmaceutical Sciences, Manipal Academy of Higher Education, Manipal, India. \\ ${ }^{2}$ Department of Cardiology, Kasturba Medical College, Manipal Academy of Higher Education, Manipal, India. \\ ${ }^{3}$ Department of Pharmacology, Melaka Manipal Medical College, Manipal Academy of Higher Education, Manipal, India.
}

\section{ARTICLE INFO \\ Received on: 13/02/2020 \\ Accepted on: 26/04/2020 \\ Available online: 05/06/2020}

Key words:

Angiotensin II receptor

blocker, hyperkalemia,

hyponatremia, hypertension,

renin-angiotensin-

aldosterone system.

\begin{abstract}
Angiotensin II receptor blockers (ARBs) are the antihypertensive drugs associated with side effects, majorly such as cough and electrolyte disturbance. Azilsartan is a newly marketed ARB in India, not even having a well-established adverse effect profile. Here, we present a 58-year-old female who was admitted to the emergency department showing signs and symptoms of delirium, disorientation, and vomiting for 4 days. The patient is known to have coronary artery disease so that she underwent coronary artery bypass grafting. She was recently started on azilsartan for her chronic hypertension, along with other drugs. The presence of electrolyte imbalance in laboratory reports and current symptoms suggested azilsartan-induced encephalopathy. The patient was recovered after discontinuation of azilsartan. This case enlightens the clinical characteristics, possible mechanism, and treatment strategy opted to correct the condition.
\end{abstract}

\section{INTRODUCTION}

Encephalopathy is an acute inflammation of the brain parenchyma. Metabolic encephalopathy is a diverse category that describes abnormalities of electrolytes, vitamins, water retention, and the other chemicals related to the brain functions (Berisavac et al., 2017).

Azilsartan is a newly launched antihypertensive drug that belongs to the angiotensin II receptor blockers (ARB) class. ARBs involve in selective blocking of angiotensin II to $\mathrm{AT}_{1}$ receptors, therefore causing an increase in vasodilation and a decrease in aldosterone effects (Kurtz and Kajiya, 2012; Zaiken and Cheng, 2011). However, the side effect profile of traditional ARBs is quite significant, including hyperkalemia (Bandak et al., 2017). In our opinion, this is the first case enlightening the adverse effect profile of azilsartan in a hypertensive patient. This case represents

\footnotetext{
*Corresponding Author

Kanav Khera, Department of Pharmacy Practice, Manipal College of Pharmaceutical Sciences, Manipal Academy of Higher Education, Manipal 576104, India.E-mail: kanav.khera@manipal.edu
}

the clinical course, treatment, and prognosis of azilsartan-related metabolic encephalopathy.

\section{CASE PRESENTATION}

A 58-year-old female presented to the emergency department of a tertiary care center with the chief complaints of vomiting and disorientation and altered sensorium. A detailed history revealed that the patient had generalized fatigability, nausea, and multiple episodes of vomiting for 1 week. She also had reduced appetite and irregular bowel movements for a few weeks. On physical examination, the patient was restless, but there was no neck stiffness; Kernig's and Brudzinski's signs were negative.

The patient had a history of hypertension and diabetes mellitus for more than 5 years. Four years back, she had a non-STelevation myocardial infarction, where the left anterior descending artery (LAD) was diseased. She underwent percutaneous coronary intervention (PCI) with drug-eluting stent to LAD. An Echocardiogram (ECHO) was showing normal left ventricle (LV) function. The patient was started on antiplatelet drugs, i.e., aspirin (loading dose of $325 \mathrm{mg}$ and then $150 \mathrm{mg}$ once daily as a maintenance dose), clopidogrel (loading dose of $300 \mathrm{mg}$ and then $75 \mathrm{mg}$ once daily as a maintenance dose), along with atorvastatin 
Table 1. The course of abnormal laboratory parameters from admission to discharge.

\begin{tabular}{lcccccccc}
\hline \multicolumn{1}{c}{ Parameters } & \multicolumn{7}{c}{ Days from admission } \\
& 1st & 2nd & 3rd & 4th & 5th & 7th & 9th & 11th \\
\hline Serum creatinine (mg/dl) & 1.6 & 1.7 & 2.1 & 1.8 & 1.6 & 1.4 & 1.4 & 1.3 \\
Sodium (mmol/l) & 117 & 120 & 121 & 128 & 131 & 129 & 134 & 142 \\
Potassium (mmol/l) & 5.9 & 6.2 & 5.5 & 5.5 & 4.7 & 3.9 & 3.7 & 3.3 \\
Urea (mg/dl) & 59 & 69 & 83 & 82 & 76 & 69 & 46 & 32 \\
Hemoglobin (g/dl)/ hematocrit (\%) & $10 / 29.8$ & & $9.2 / 29.0$ & & $8.7 / 28.4$ & $8.3 / 27.6$ & $7.4 / 26.1$ & $8.1 / 27.2$ \\
TLC (cells/mm $\left./ \mathrm{mm}^{3}\right)$ & 11.3 & & 12.1 & & 10.2 & 10.0 & 9.8 & 9.4 \\
\hline
\end{tabular}

TLC: total leukocyte counts.

of $40 \mathrm{mg}$ per day, antihypertensive agents (ramipril $2.5 \mathrm{mg}$ once daily and metoprolol extended release $25 \mathrm{mg}$ once daily), and antihyperglycemic drug (glimepiride $1 \mathrm{mg}$ daily).

One year back, 3 years after LAD-PCI, she again present with acute pulmonary edema and angina, and a coronary angiogram showed calcific left main coronary artery and double vessel disease with left circumflex and LAD restenosis. At this admission, she underwent coronary artery bypass grafting with three grafts. ECHO showed mild-to-moderate LV dysfunction with ejection fraction (EF) of $44 \%$. The patient was continued on aspirin with atorvastatin and newly added ivabradine, isosorbide dinitrate, and hydralazine combination for LV dysfunction. Glimepiride 1 mg daily was continued for diabetic status. After a few months of this admission, the patient stopped the medicines for 1 week and developed acute shortness of breath, progressive in nature. She admitted again, and ECHO showed a severe LV dysfunction with EF 20\% and moderate-to-severe mitral regurgitation (MR), severe pulmonary arterial hypertension $(\mathrm{PAH})$, and right ventricle dysfunction. The patient was treated with a diuretic, oxygen therapy, and inotropes for heart failure. The high blood sugar levels were managed with insulin and oral hypoglycemic agents (glimepiride $1 \mathrm{mg}$ once daily). Ramipril $2.5 \mathrm{mg}$ once daily and metoprolol extended release $25 \mathrm{mg}$ once daily were continued for hypertensive history and current high blood pressure.

One month back, the patient came for the follow-up to the outpatient department, where her BP was $>140 / 90 \mathrm{mmHg}$, and ramipril $2.5 \mathrm{mg}$ was substituted by azilsartan $40 \mathrm{mg}$ orally twice daily. At the present admission, the patient presented with the above mentioned signs and symptoms. The laboratory investigations were done, which showed deranged renal parameters. Liver enzymes and thyroid-stimulating hormone were normal. Hematological parameters show low hemoglobin and hematocrit and slight high total leukocyte counts (TLCs) (Table 1).

Azilsartan was stopped in view of symptoms and deranged electrolytes and renal parameters. A neurology consultation was sought who opined nil intervention. Magnetic resonance imaging of brain was normal. A nephrologist suggested the initial stage of cardiorenal syndrome, which was improved later. Hyponatremia and hyperkalemia were improved within 1 week. ECHO showed a moderate LV dysfunction and moderate MR along with moderate $\mathrm{PAH}$. The patient was discharged on the previous combination of aspirin, clopidogrel, and statin with the same dose and frequency, teneligliptin $20 \mathrm{mg}$ once daily (switched from glimepiride because of an acute rise in creatinine level), and carvedilol $3.125 \mathrm{mg}$ once daily (azilsartan was stopped, and metoprolol was switched to carvedilol based on the LV dysfunction). The patient was doing well on a 30day follow-up, and the electrolyte reports and renal parameters were in the normal range. Hemoglobin was also noted more than $10 \mathrm{~g} / \mathrm{dl}$.

\section{DISCUSSION}

Encephalopathy is any diffuse disease of the brain, which alters the structure or function of the brain. Diverse etiologies are discovered, including infectious agents, tumor, increased exposure to toxic elements (such as certain metals and drugs), trauma, and cerebral ischemia. Neurological symptoms may include progressive loss of memory and cognitive ability, nystagmus, and seizures. One of the complications of encephalopathy is metabolic encephalopathy (Butterworth and Layrargues, 1990). Few cases have shown an increase in serum ammonia levels to patients on ARB treatment, due to the reduced renal excretion. Serum ammonia levels are a direct indication of hepatic encephalopathies. Hepatic encephalopathy is one of the causes of metabolic encephalopathy (Ong et al., 2003).

Metabolic encephalopathy can be defined as a potentially reversible abnormality of the brain function caused by processes of extracerebral origin. The most common symptom is delirium. However, mood and orientation disorder thought, memory disorder, intellectual deterioration, dementia, and depression may also occur. Metabolic encephalopathy can also include drug ingestions or medication side effects that affect the chemical transmitters in the brain. If left untreated, however, it may result in secondary structural damage to the brain (Fraser and Arieff, 1997). Still, the pathophysiological mechanisms have not been completely understood. However, an inflammation could trigger the endothelial activation in the brain, which leads to the malfunctioning of the blood-brain barrier and could result in the activation of cytokines and chemokines, which enter and damage the cellular metabolism. This initiates the mitochondrial dysfunction as well as oxidative stress, resulting in the disruption of neurotransmission and apoptosis (Tan et al., 2019). ARBs can be thought to have similar actions as they, too, disrupt the neurotransmission (Raebel, 2012). Metabolic encephalopathies are of two major types - those due to the lack of glucose, oxygen, or metabolic cofactors and those due to peripheral organ damage. Low-grade fever, anemia, mild-sedative intoxication, and minimal renal dysfunction are usually found in various combinations (Berisavac et al., 2017). Anemia, hyperkalemia, and hyponatremia 
were noted in the present patient also, which indicate the metabolic disturbances.

Azilsartan is distinguished from other sartans in the matter of efficacy and 24-hour blood pressure control (Kurtz and Kajiya, 2012). Similar to losartan, an $\mathrm{AT}_{1}$ receptor antagonist azilsartan inhibits the augmentation of noradrenergic neurotransmission, sympathetic tone enhancement, and biological effects of angiotensin-II such as vasopressin release, pressor responses, and the release of aldosterone and adrenal catecholamines. Azilsartan is also selective and potent (Das et al., 2015). In this case presentation, the patient might develop the sign and symptoms by the same mechanism. Azilsartan as an ARB can develop hyperkalemia as its side effect and induces an electrolyte imbalance (Georgiopoulos et al., 2016). The involvement of the renin-angiotensin-aldosterone system increases the serum of potassium levels by interfering with aldosterone secretion mediated by angiotensin II (Pradhan et al., 2019; Weir and Rolfe, 2010). Hyperkalemia could cause hyperexcitation of the neurons and, hence, could be the cause of deliriums. According to a study conducted in Guangxi Medical University, China, hyperkalemia could also ameliorate a brain injury by alleviating calcium overload, inhibiting the activity of NCX1, and lowering the concentration of $\mathrm{Ca}^{+}$(Tan et al., 2019).

Hyponatremia caused by azilsartan may be explained by reduced aldosterone release and renal tubular sodium reabsorption mediated by angiotensin II (produced by AT1 receptor inhibition) (Das et al., 2015). This case did not present any evidence of adrenaline insufficiency, hypothyroidism, or established cardiorenal syndrome, which augments the condition to metabolic disturbances in the brain; there is a clear indication that the metabolic encephalopathy was induced by azilsartan use and the discontinuation of the drug improved it.

\section{CONCLUSIONS}

The current case suggests that azilsartan is associated with metabolic encephalopathy caused by electrolyte imbalance in a chronic hypertensive patient. The patient was initiated on a high dose of azilsartan, and starting with a low dose may help in avoiding such conditions. Metabolic encephalopathy is reversible in the primary stage and, therefore, should not be allowed to progress, which would cause the secondary structural changes to the brain. The elderly patients should be followed thoroughly to notice any imbalances and rectify immediately.

\section{ACKNOWLEDGMENT}

None.

\section{STATEMENT OF ETHICS}

Ethical approval (informed consent) was received for publication before preparing the manuscript.

\section{CONFLICT OF INTEREST}

None of the authors declare any conflict of interest.

\section{REFERENCES}

Bandak G, Sang Y, Gasparini A, Chang AR, Ballew SH, Evans $\mathrm{M}$, et al. Hyperkalemia after initiating renin-angiotensin system blockade: The Stockholm Creatinine Measurements (SCREAM) project. J Am Heart Assoc, 2017; 6(7):e005428.

Berisavac II, Jovanović DR, Padjen VV, Ercegovac MD, Stanarčević PD, Budimkić-Stefanović MS, et al. How to recognize and treat metabolic encephalopathy in Neurology intensive care unit. Neurol India, 2017; 65(1): 123 .

Butterworth R, Layrargues G. Hepatic encephalopathy. Humana Press, Totowa, NJ, 1990.

Das S, Bandyopadhyay S, Ramasamy A, Prabhu VV, Pachiappan S. A case of losartan-induced severe hyponatremia. J Pharmacol Pharmacother, 2015; 6(4):219.

Fraser CL, Arieff AI. Epidemiology, pathophysiology, and management of hyponatremic encephalopathy. Am J Med, 1997; 102(1):67-77.

Georgiopoulos G, Katsi V, Oikonomou D, Vamvakou G, Koutli E, Laina A, et al. Azilsartan as a potent antihypertensive drug with possible pleiotropic cardiometabolic effects: a review study. Front Pharmacol, 2016; $7: 235$

Kurtz TW, Kajiya T. Differential pharmacology and benefit/ risk of azilsartan compared to other sartans. Vasc Healt Risk Manag, 2012; $8: 133$

Ong JP, Aggarwal A, Krieger D, Easley KA, Karafa MT, Van Lente $\mathrm{F}$, et al. Correlation between ammonia levels and the severity of hepatic encephalopathy. Am J Med, 2003; 114(3):188-93.

Pradhan A, Tiwari A, Sethi R. Azilsartan: current evidence and perspectives in management of hypertension. Int J Hypertension, 2019; 2019:1-8. doi:10.1155/2019/1824621

Raebel MA. Hyperkalemia associated with use of angiotensinconverting enzyme inhibitors and angiotensin receptor blockers. Cardiovasc Ther, 2012; 30(3):e156-66.

Tan XF, Qin T, Li N, Yang YG, Zheng JH, Xie L, Chen MH. High-potassium preconditioning enhances tolerance to focal cerebral ischemia-reperfusion injury through anti-apoptotic effects in male rats. J Neurosci Res, 2019; 97(10):1253-65.

Weir MR, Rolfe M. Potassium homeostasis and reninangiotensin-aldosterone system inhibitors. Clin J Am Soc Nephrol, 2010; 5(3):531-48.

Zaiken K, Cheng JW. Azilsartan medoxomil: a new angiotensin receptor blocker. Clin Ther, 2011; 33(11):1577-89.

How to cite this article:

Sekhar AG, Singh A, Chauhan S, Khera K, Devasia T. Azilsartan causing metabolic encephalopathy. J Appl Pharm Sci, 2020; 10(06):123-125. 